\title{
Etching Effect on Microstructural Behavior of Gas Atomized Al-20 mass\% Si Alloy Powder
}

\author{
Soon-Jik Hong \\ Division of Advanced Materials Engineering \& Institute for Rare Metals, Kongju National University, \\ 275, Budae-dong Cheonan City, Cheongnam 330-717, Korea
}

In this research, a metallographic investigation of as atomized Al-20 mass $\% \mathrm{Si}$ alloy powders, etched at surface and cross-section, was conducted by scanning electron microscopy. The surface micrographs of the etched powders showed the existence of primary Si and eutectic Si on the microstructure of the powder surface, and satellite particles adhering to the larger particles, while it was impossible to observe the surface microstructure in the case of the non-etched powder surface. The coarse primary Si phases in these powder particles were not present, indicating that their formation was effectively suppressed by the rapid solidification. In addition, it was possible to observe the microstructure of satellite powder surface such as fine cellular or featureless structure due to surface etching. [doi:10.2320/matertrans.M2010008]

(Received January 8, 2010; Accepted March 1, 2010; Published April 25, 2010)

Keywords: aluminum-silicon powder, gas atomization, etching, microstructure, satellite powder

\section{Introduction}

Al-Si alloys are used in many areas of application such as the automotive and electronic industries due to their good wear resistance and low thermal expansion coefficient. These properties are attributed to the high volume fraction of hard Si particles embedded in the Al-matrix. ${ }^{1,2)}$ In order to improve the mechanical properties, the distribution and size of the silicon particles in the alloy, has been identified as the key parameter. A lot of techniques such as modification, ${ }^{3,4)}$ ternary alloying, ${ }^{5)}$ spray-deposition, ${ }^{6)}$ or rapid solidification processing $^{7)}$ have been applied to refine the primary $\mathrm{Si}$ crystals and achieve their homogeneous distribution in the hypereutectic Al-Si alloys. Of all the above mentioned processes, rapid solidification of metallic metals has been known to produce altered constitutional effects such as the formation of supersaturated solid solutions, metastable intermetallic phases and even amorphous alloys. ${ }^{8-10)}$ In gas atomization process, the superheated molten material is sprayed in the form of droplets, which solidifies during their flight into the chamber. The microstructural changes of Al-Si alloy system powders through the gas atomization process have been of great interest in the last fifteen years. In most of the researches, Al-Si alloy has been rapidly solidified and its microstructural features were characterized by OM, SEM after etching with Keller's reagent. In addition, all the microstructural analyses were conducted from the crosssectional viewpoint of the rapidly solidified powders. However, no other researches have been reported the surface analysis of the rapidly solidified powders by etching the surface using $\mathrm{KOH}$ or any other reagents. In this study, the Al-20 mass\% Si binary alloy system was chosen to investigate the microstructural behavior of the gas atomized powders with etching effect on the surface and cross-sectional microstructure by metallographic investigation in order to avoid the interference of ternary intermetallics present in higher order alloy systems.

\section{Experimental Procedure}

Gas atomized Al-20 mass\%Si alloy powders were synthe- sized by melting the master alloy in a graphite crucible $200 \mathrm{~K}$ above the melting temperature, and bottom pouring through a boron nitride melt delivery nozzle of $3 \mathrm{~mm}$ diameter into a confined $\mathrm{N}_{2}$ gas atomizer operating at a pressure of 1.4 MPa. The size distribution of the alloy powder particles was measured by conventional mechanical sieving method and powders with a specific size range of $45 \sim 150 \mu \mathrm{m}$ were chosen for this investigation. For metallographic observation, samples were cold-mounted, mechanically polished, crosssection and surfaces were etched with Keller's and $\mathrm{KOH}$ reagents, respectively, and finally, observed by scanning electron microscopy (SEM). For SEM observation, samples were deeply etched and coated with a thin layer of gold to avoid the charging effect. The composition of the surface microstructure was determined by the Energy dispersive X-ray spectroscopy (EDX).

\section{Results and Discussion}

Figure 1(a) shows the typical surface of the gas atomized powders, where many satellite particles can be observed at a higher magnification on the coarser powders surface. In the large atomized powders, a substantial agglomeration of the smaller particles was observed along with numerous small satellite particles (dot circles), which were $\sim 15 \mu \mathrm{m}$ in diameter and adhered to the large particles. In addition, it was impossible to observe any dominating microstructures such as primary $\mathrm{Si}$ and eutectic $\mathrm{Si}$ on the surface of as gasatomized Al-20 mass $\% \mathrm{Si}$ powders due to the presence of thick oxide film as shown in Fig. 1(a).

The observation of atomized powder surface after etching shows very interesting result. The surface micrographs of the etched powder (Fig. 1(b)) showed a primary Si and eutectic $\mathrm{Si}$ on the microstructure of the powder's surface, and satellite particles adhering to the larger particles, while it was impossible to observe the surface microstructure in case of the non-etched powder surfaces (Fig. 1(a)). In order to observe a more detailed microstructure, the etched powder surface was observed at a higher magnification by SEM as depicted in Fig. 2(a). The surface microstructure of the powder consists of primary and eutectic Si crystals embedded 


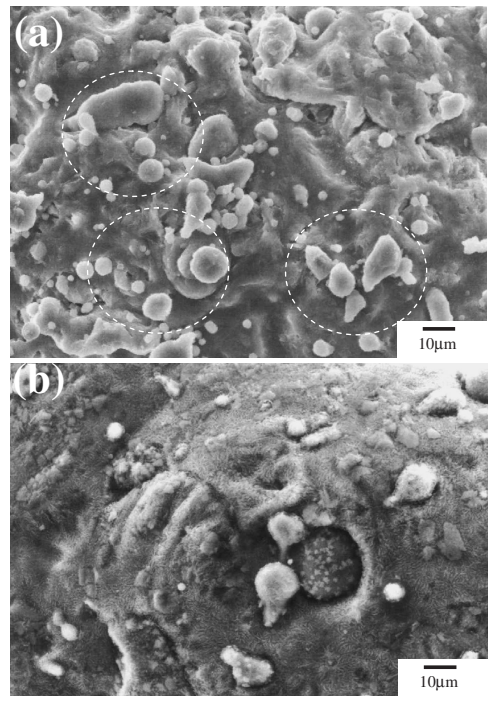

Fig. 1 SEM micrographs showing powder surface before $\mathrm{KOH}$ etching (a) and $\mathrm{KOH}$ etched powder surface (b) of gas-atomized Al20 mass $\%$ Si alloy powders.

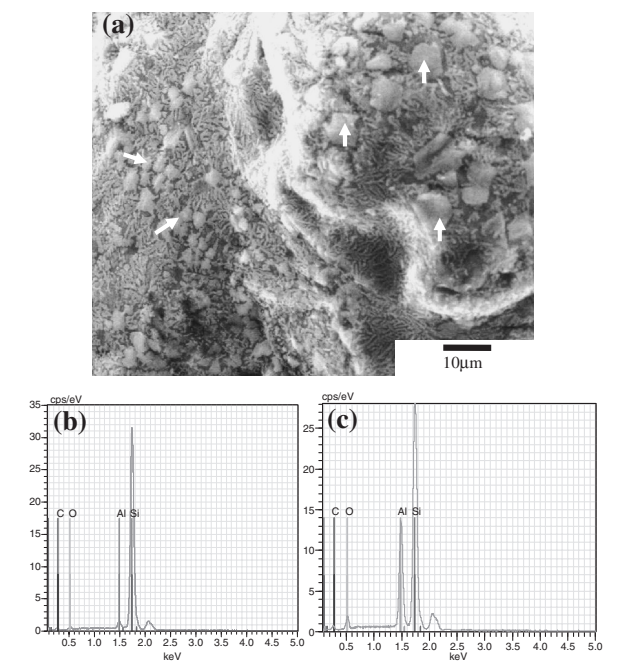

Fig. 2 SEM micrograph of the etched powder surface at higher magnifications (a) and the EDX analysis for primary $\mathrm{Si}(\mathrm{b})$ and $\mathrm{Al}-\mathrm{Si}$ eutectic structure (c) of as gas-atomized Al-20 mass\%Si powders.

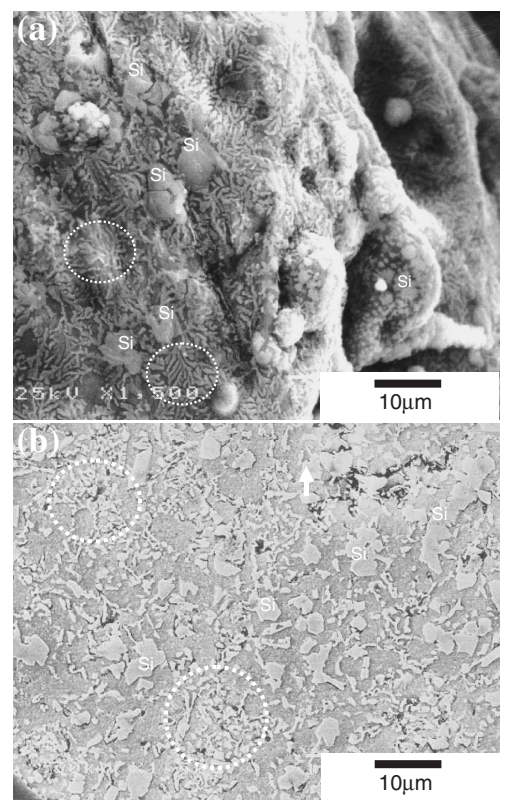

Fig. 3 SEM microstructures of gas atomized Al-20 mass $\%$ Si powder; $\mathrm{KOH}$ etched powder surface (a), cross-sectional microstructure. in the aluminum matrix. The typical size of primary $\mathrm{Si}$ as marked by arrows is about $\sim 10 \mu \mathrm{m}$. It means that the etching of as atomized powder was effective on the observation of surface microstructure from the gas atomized powders. Although a lot of researches were carried out to observe the cross-sectional microstructure of Al-Si alloy powders, however, none of those revealed the surface microstructures. To ensure the formation of primary and eutectic microstructures and to reveal their concentrations, the EDX analysis was performed and the results are demonstrated in Fig. 2(b) and (c). The composition of primary Si (Fig. 2(b)) was obtained as 89.5 mass $\% \mathrm{Si}$ and 2.06 mass $\% \mathrm{Al}$ (including $\mathrm{C}=6.53$ mass $\%$ and $\mathrm{O}=2.03$ mass $\%)$. Whereas in case of the eutectic Al-Si structure (Fig. 2(c)), the $\mathrm{Si}$ and $\mathrm{Al}$ concentration was found to be 69.32 mass $\%$ and 18.19 mass $\%$ (including $\mathrm{C}=7.27$ mass $\%$ and $\mathrm{O}=5.23$ mass $\%$ ), respectively. The lower quantity of $\mathrm{Al}$ in the eutectic was due to the melting of $\mathrm{Al}$ in response to the etching by the $\mathrm{KOH}$ reagent. In order to compare the microstructures between the surface and cross-section of the powders, two specimens were prepared as shown in Fig. 3.

Figure 3 shows high magnification SEM micrograph of the as atomized Al-20 mass\%Si alloy powders from etched powder surfaces (a) and cross-sections (b) showing primary $\mathrm{Si}$ and eutectic Si in different size and shape. The etched powder surface (Fig. 3(a)) shows granular, block or flat-like primary $\mathrm{Si}$ with the average size of $2-7 \mu \mathrm{m}$ and is much finer size than that of the cross-sectional microstructure due to high solidification rate at the surface during atomization. In addition, the surface of primary Si shows parabolic shape according to their originally solidified structure. The microstructure of eutectic Si in the etched powder surface shows a dendritic morphology while cross-sectional microstructure shows partially broken and distributed particles. The crosssectional microstructure of the powders (Fig. 3(b)) consists of flat silicon particles with irregular and sharp edgy shape compared to the surface etched powders due to cutting and polishing of the primary Si particles.

During atomization, powder particle of various sizes collides with each other in the gas turbulence. For example; during collision, the larger particles might still be in liquid or semi-solid state, while smaller particles would have been transformed into a fully solid state as shown in Fig. 4. At the time of their flight in the atomization chamber, there is a possibility adhering of the fully-solidified smaller particles (powder A) to the larger liquid or semi-solid particles (powder B). Figure 4(a) shows the cellular structure and a cellular-dendritic structure indicating high solidification rate as represented by the dot circles. The structural zones in the atomized powders can generally be classified into a featureless zone, a fine cellular zone and a cellular-dendritic zone according to the thermal history experienced during solidification.

Figure $4(\mathrm{~b})$ shows a very fine $(6.4 \mu \mathrm{m})$ spherical satellite powder with very smooth surface; this is the typical morphology of a fine powder with diameter's less than about $10 \mu \mathrm{m}$. The smooth surface of the fine powder implies that it solidified rapidly at a very high cooling rate. It is mentionable that the optical revelation of the particles smaller than $10 \mu \mathrm{m}$ was difficult as it was extremely finer and rather irresponsive to etching. The coarse primary phases in these powder particles were not present, indicating that their formation was effectively suppressed by the rapid solidification. As the particle size decreases, the structure generally transforms into a fine cellular-dendritic structure. A further decrease in the particle size would eventually produce a fine cellular structure beside the cellular-dendritic structure as evident in Fig. 4(b).

Figure 5(a) and (b) shows a microstructural comparison between the etched surface and cross-sections of the satellite particles. It can be seen that very fine particles adhere to the larger particles to form satellites. As the satellites are 


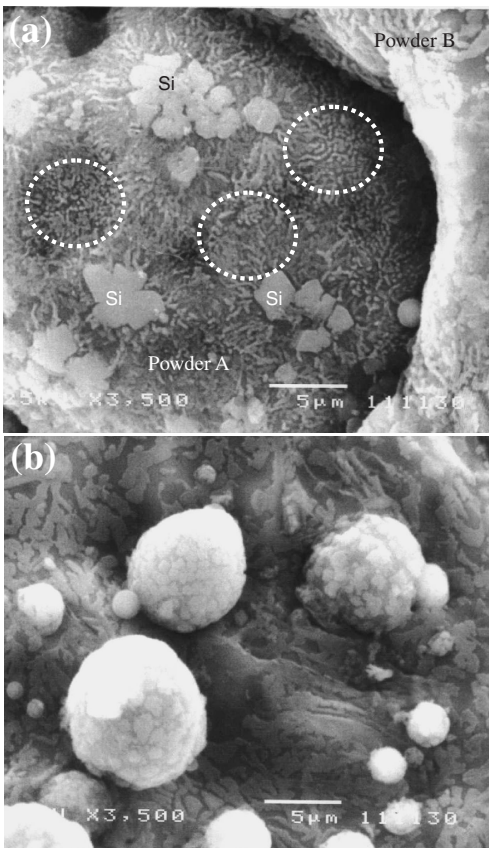

Fig. 4 SEM micrographs of Al-20 mass $\%$ Si alloy powder surface after $\mathrm{KOH}$ etching showing the adhered satellite powder particles on the coarse powder (a) and very fine satellite powder particles (b) on the coarse powder.

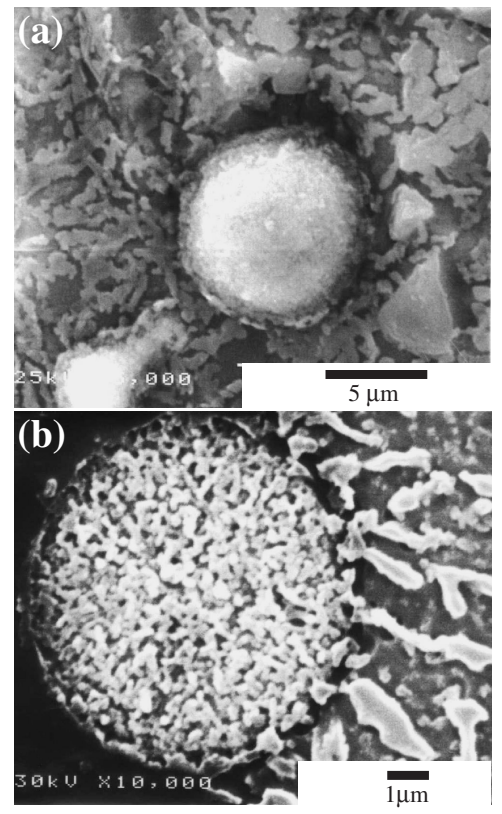

Fig. 5 SEM micrographs of adhered satellite powder particle on the coarse powder after $\mathrm{KOH}$ etching (a) and cross-sectional microstructure (b) of the satellite powder particles.

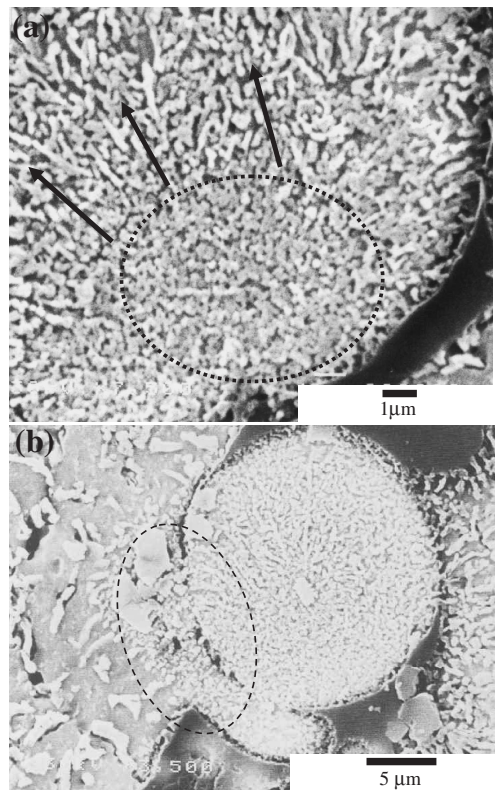

Fig. 6 SEM micrographs showing solidification direction (a) from the edge into the center of powder with solidification rate during gas atomization and the interface (b) between coarse particle and the adhered small particle. nearly a perfect spheroidal, it is thought that collisions happened when the satellite particles were almost solidified. Figure 5(a) shows the droplets enveloping more than half of the periphery of the powder particles being capped. The featureless microstructure of the splat caps suggests that the particles being capped had already been chilled when the collisions occurred.

Figure 5(b) shows the cross-sectional micrograph of the adhered fine powder particle on a large particle. The primary and eutectic $\mathrm{Si}$ of the satellite powders are very small compared to that of the large particle. The shape of the primary $\mathrm{Si}$ in the coarse powder is trapezoidal in nature. However, the primary and eutectic Si of the adhered smaller particles are fine and homogeneously distributed in the Al matrix so that it was impossible to distinguish between the primary $\mathrm{Si}$ and eutectic $\mathrm{Si}$. Moreover, the primary and eutectic Si of the adhered satellite particles were spherical/ polygonal shape instead of the chain-like networks observed in the coarse powder.

The inhomogeneous microstructure in the powders is generally caused by the varying undercooling effect across the powder particle sections, which resulted in the operation of different solidification mechanism. Therefore, all the solidified droplets showed a multiple and complex microstructure, and are generally consist of eutectic, dendritic and microcellular morphologies as observed in Fig. 5(a) and (b). In addition, the smaller droplet cools faster resulting in fine spacings to form the cellular structures.

Figure 6(a) shows the cross-sectional micrograph of the solidification behavior and its propagation from the surface into the matrix. Each equiaxed cell usually contains several intercellular dispersoids. It is of particular interest to see the dendritic configuration of the dispersoids at the edge of the powder surface in Fig. 6(a), which clearly illustrates the occurrence of a structural change from granular dispersoid to dendritic ones along with the decrease in undercooling during solidification as indicated by dotted circles. The eutectic microstructure and the spacing between them gradually increase at larger distances in respect to the location of the nucleation site due to the difference in cooling effect (marked by arrows). This cooling effect further influences the morphological changes from microcellular to dendritic and to the eutectic structure. Hence, it implies that the initial solidification around the nucleation sites was so rapid that the early formation of dispersoids during solidification was suppressed there.

Figure 6(b) shows the high-magnification SEM micrograph of the interface between the coarse particle and the adhered satellite particle (marked as the dotted circle). The interface shows a finer microstructure comparing to the inner section of the coarse powders. This may be due to the higher solidification rate at the interface aided by the already solidified fine particle. The existence of the already solidified fine particle acts as a precursor to further increase the solidification rate of the coarse particle after their collision.

The gas atomization process usually results in a wide range of particle sizes due to the variation in the cooling rate, which is inversely proportional to the size of the liquid droplets. During atomization the heat from the coarser droplet is extracted by the atomizing gas through the convection process. However, as soon as this coarser particle collides with the already solidified smaller particles, the heat extraction process transforms into the conduction process resulting in a faster heat extraction from the collided portion of the coarser droplet to the smaller much cooler solidified particles. Such kind of phenomena brings the collided portion 
of the coarser droplet to a greater degree of undercooling ${ }^{11)}$ and facilitates heterogeneous nucleation at the collided interface. As the solidification proceeds and the liquid-solid interface rapidly moves forward in a planar manner, a large amount of latent heat is concurrently released. Such liberation of latent heat causes the temperature of the liquid-solid interface to increase and thus, reducing the undercoolings known as recalescence, affects the solidification rate throughout the powder particle sections. ${ }^{12,13)}$ It is expected that an improvement in the design parameters and process parameters of gas atomization would minimize the possibility of collisions between the particles. Though it is interesting to find out the direct relationship between the collisions and mechanical properties, such complex phenomena are yet to be determined in the future works.

\section{Conclusions}

The microstructure of Al-20 mass $\%$ Si alloy powder was investigated as a function of the powder etching condition such as surface and cross-sectional. SEM micrograph of the as atomized Al-20 mass $\% \mathrm{Si}$ alloy powders from etched powder surfaces and cross-sections showed different primary $\mathrm{Si}$ and eutectic $\mathrm{Si}$ in size and shape. The etched powder surface showed granular, block or flat-like primary Si with an average size of $2-7 \mu \mathrm{m}$ and was much finer size than that of cross-sectional microstructure due to high solidification rate at the surface during atomization. The surface of primary $\mathrm{Si}$ showed parabolic shape according to their originally solidified structure. The microstructure of eutectic Si in the etched powder surface showed a dendritic morphology, while crosssectional microstructure showed partially broken and distributed particles. In addition, the primary and eutectic Si of the adhered satellite particle was in spherical/polygonal shape instead of the flat-like networks observed in the coarse powder. Finally, the surface etching of as atomized powder was effective on the observation of surface microstructure from the gas atomized powders.

\section{Acknowledgment}

This work was supported by the Korea Research Foundation Grant funded by the Korean Government (MOEHRD, Basic Research Promotion Fund) (KRF-331-2007-1D00577).

\section{REFERENCES}

1) J. Clarke and A. D. Sarkar: Wear 54 (1979) 7.

2) T. K. A. Jaleel, N. Raman, S. K. Biswas and K. S. S. Murthy: Aluminum 60 (1984) 493.

3) M. D. Hanna, S. Lu and A. Hellawell: Metall. Trans. A 15A (1984) 459-469.

4) G. Purcek, O. Saray and O. kul: Met. Mater. Int. 16.1 (2010) 145-154.

5) A. Knuutinen, K. Nogita, S. D. McDonald and A. K. Dahle: J. Light Met. 1 (2001) 229-240.

6) J. Zhou, J. Duszczyk and B. M. Korevaar: J. Mater. Sci. 26 (1991) 5257-5291.

7) S. J. Hong, T. S. Kim, W. T. Kim and B. S. Chun: Mater. Sci. Eng. A 226-228 (1997) 878-882.

8) Y. H. Kim, A. Inoue and T. Masumoto: Mater. Trans., JIM 32 (1991) 331.

9) S. J. Hong, T. S. Kim, H. S. Kim, W. T. Kim and B. S. Chun: Mater. Sci. Eng. A 271 (1999) 469-476.

10) H. Jones: Non-equilibrium Processing of Materials, ed. by C. Suryanarayana, Pergamon Press, Oxford, UK, (1999) pp. 23-45.

11) H. C. Neubing: Powder Metal 25 (1982) 160.

12) C. G. Levi and R. Mehrabian: Metall. Trans. A 13A (1982) 13.

13) G. J. Marshall: J. Mater. Sci. 22 (1987) 3581. 